\title{
Correction to: A newly noninvasive model for prediction of non-alcoholic fatty liver disease: utility of serum prolactin levels
}

Pengzi Zhang ${ }^{1+}$, Wenhuan Feng ${ }^{1 \dagger}$, Xuehui $\mathrm{Chu}^{2+}$, Xitai Sun ${ }^{2}$, Dalong $\mathrm{Zhu}^{1}$ and Yan Bi ${ }^{{ }^{*}}$

\section{Correction to: BMC Gastroenterol \\ https://doi.org/10.1186/s12876-019-1120-z}

Following publication of the original article [1], we have been notified that the given name of one of the authors was spelled incorrectly. It is now Wenghuan Feng and should be as follows:

Wenhuan Feng

The original article has been corrected.

\section{Author details}

'Department of Endocrinology, Drum Tower Hospital Affiliated to Nanjing University Medical School, No 321, Zhongshan Road, Nanjing 210008, Jiangsu, China. ${ }^{2}$ Department of General Surgery, Drum Tower Hospital Affiliated to Nanjing University Medical School, Nanjing, China.

Published online: 18 February 2020

\section{Reference}

1. Zhang, et al. A newly noninvasive model for prediction of non-alcoholic fatty liver disease: utility of serum prolactin levels. BMC Gastroenterol. 2019; 19:202. https://doi.org/10.1186/s12876-019-1120-z.

The original article can be found online at https://doi.org/10.1186/s12876019-1120-z

* Correspondence: biyan@nju.edu.cn

${ }^{\dagger}$ Pengzi Zhang, Wenhuan Feng and Xuehui Chu contributed equally to this work.

'Department of Endocrinology, Drum Tower Hospital Affiliated to Nanjing University Medical School, No 321, Zhongshan Road, Nanjing 210008, Jiangsu, China

Full list of author information is available at the end of the article 